\title{
Relation between the Amount of Fresh Bare Surface at the Crack Tip and the Fatigue Crack Propagation Rate
}

\author{
Masayuki SHIMOJO, Yakichi HIGO') and Shigetomo NUNOMURA') \\ Graduate School, Tokyo Institute of Technology, Nagatsuta-cho, Midori-ku, Yokohama, Kanagawa-ken, 227 Japan. \\ 1) Precision and Intelligence Laboratory (Research Laboratory of Precision Machinery and Electronics), Tokyo Institute of \\ Technology, Nagatsuta-cho, Midori-ku, Yokohama, Kanagawa-ken, 227 Japan.
}

(Received on February 21, 1991; accepted in final form on March 22, 1991)

\begin{abstract}
Under the constant potential fatigue crack was propagated in pure titanium polycrystal in $\mathrm{NaCl}$ aqueous solution which had very small effect on the fatigue crack propagation rate. The polarization current during the fatigue crack propagation was measured and the electric charge passed during one fatigue cycle was calculated. The amount of fresh bare surface produced at the crack tip during one fatigue loading was quantified by the charge, and this related linearly to the crack propagation rate. This suggests that the shapes of the crack tip show the similar profile through the tested $\Delta K$ range. By comparing with the plastic zone size and the crack tip opening displacement, it is also concluded that the fatigue crack propagation mechanism directly relates to the creation of fresh bare surface at the crack tip, not directly to the plastic zone size or the crack tip opening displacement.
\end{abstract}

KEY WORDS: fresh bare surface; fatigue crack propagation; electric charge; plastic zone size; titanium; $\mathrm{NaCl}$ aqueous solution.

\section{Introduction}

Fatigue of metallic materials is not caused by cyclic elastic deformation but requires cyclic plastic deformation. In particular, crack propagation in fatigue is believed to be caused by cyclic plastic deformation at the crack tip. As a result, the striation on the fracture surfaces, which is characterized by the fatigue crack propagation, is produced by the plastic deformation during each loading cycle at the crack tip. The striation spacing corresponds to the crack propagation rate.

A number of models of the crack propagation mechanism have been proposed. ${ }^{1-3)}$ According to these models, amount of plastic deformation at the crack tip is estimated by a crack tip opening displacement or reversed plastic zone size. However, except for measurements by direct observation with scanning electron microscope at near final fracture region, ${ }^{4,5)}$ the amount of plastic deformation, especially the fresh bare surface at the crack tip which directly relates to the crack propagation is only an estimation. Therefore, it is very important for understanding the mechanisms to measure the actual amount of fresh bare surface produced in each fatigue cycle at the crack tip.

When the fresh bare surface is generated on the specimen under the potentiostatic condition in a liquid environment, it will be corroded rapidly because of its higher activity. The amount of the corrosion can be obtained electrochemically by measuring the polarization current. Specifically on the passive metals, corrosion rate at the surface except the fresh bare surface is so low that the information on the surface other than fresh bare surface seems to be negligible. Therefore, it is concluded that almost all of the electric charge is consumed to passivate the fresh bare surface. Furthermore, the thickness of the passive film becomes constant under the fixed environment. Therefore the area of fresh bare surface can be calculated by converting the total electric charge to the passivated area. Applying this method, measurement of the production of bare surface at the crack tip during one fatigue cycle is considered possible.

In this study, fatigue crack was propagated in titanium polycrystal in an aqueous solution which has a very small effect on the fatigue crack propagation rate. ${ }^{6,7}$ Subsequently the amount of bare surface produced at the crack tip during one fatigue cycle was measured by the electrochemical method, and the results were discussed by comparison with crack propagation rate and stress intensity factor range.

\section{Experimental Procedure}

The tensile test was carried out to obtain the relation between the amount of newly produced bare surface of slip steps and the electric charge.

The tensile specimens were machined from hot-rolled and annealed poly-crystalline pure commercial titanium plate. The chemical composition of the material is shown in Table 1. The tensile direction coincides with the rolling 
Table 1. Chemical composition of the titanium plate. (wt \%)

\begin{tabular}{cccccc}
\hline $\mathrm{Fe}$ & $\mathrm{O}_{2}$ & $\mathrm{~N}_{2}$ & $\mathrm{C}$ & $\mathrm{H}_{2}$ & $\mathrm{Ti}$ \\
\hline 0.072 & 0.232 & 0.006 & 0.005 & 0.0004 & balance \\
\hline
\end{tabular}

(a)
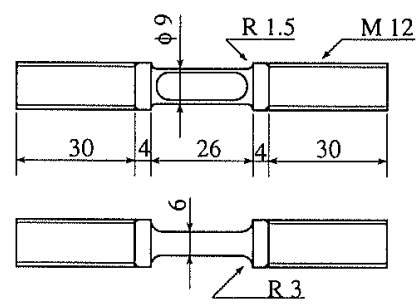

(b)
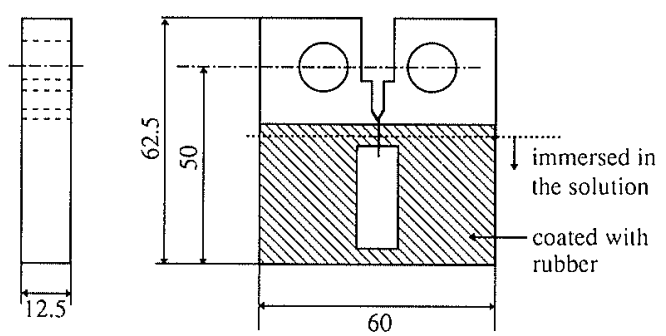

Fig. 1. Dimensions of tensile specimen (a), and CT specimen (b) employed.

direction. A strain gauge was attached on the back surface of the specimen, and then except for the front surface the specimens were coated with silicon rubber for the measurement of electric charge. The surface was finished by $1 \mu \mathrm{m}$ diamond abrasive.

CT specimens $(W=50 \mathrm{~mm}, B=12.5 \mathrm{~mm})$ for the fatigue crack propagation test were machined from $L-T$ direction of the same titanium plate as the tensile specimen. To remove the effects of the solution surface fluctuation on the specimen, the specimen was coated with rubber except around the area of crack propagation.

Both of the tensile and the fatigue test environment were aerated $0.9 \% \mathrm{NaCl}$ aqueous solution at room temperature. The potential of the specimen was kept constant at $0.1 \mathrm{~V}$ (vs. $\mathrm{Ag} / \mathrm{AgCl}$ ) by the potentiostat, which is in the passive region.

Figures 1(a) and 1(b) show the shapes of the tensile and CT specimens, respectively.

\subsection{Tensile Test}

The tensile tests were carried out under constant strain rate of about $0.05 \mathrm{sec}^{-1}$ by a closed loop servo hydraulic fatigue machine. The tensile strain was applied up to a preset strain, then tensile test was interrupted for $20 \mathrm{sec}$ until polarization current settled down to the steady state value. The tensile process was performed until total plastic strain was up to approximately 0.13 on each specimen. The preset plastic strain values were varied in a range of 0.005 to 0.13 . These tests were carried out on 10 tensile specimens.

The recorded data of polarization current and the strain in a digital memory were analyzed to obtain the relation between the amount of bare surface and the

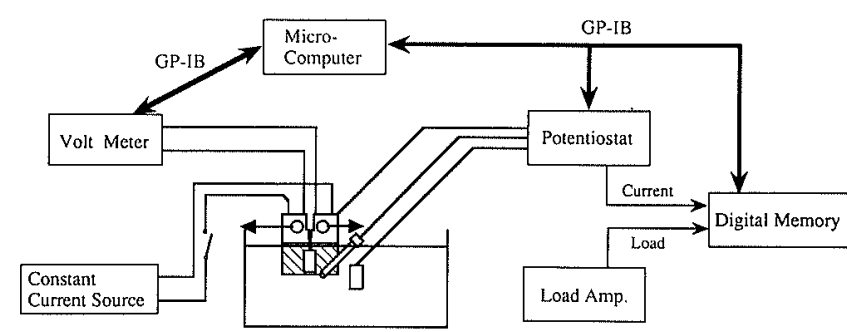

Fig. 2. Block diagram of measurement system for fatigue crack propagation and polarization current.
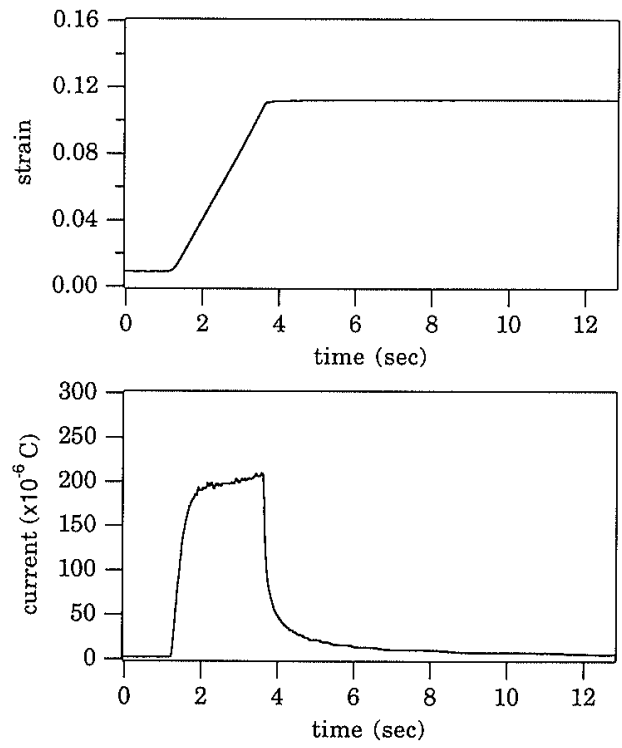

Fig. 3. Typical results of the relation between time and tensile strain (a), and also polarization current (b).

electric charge.

\subsection{Fatigue Crack Propagation Test}

Figure 2 schematically shows the block diagram of the measurement system.

Crack propagation tests were carried out with a closed loop servo hydraulic fatigue machine. Sinusoidal wave was employed in load control and the stress ratio, $R$, was 0.5 where little effect of closure was expected. Loading frequencies used were $0.3,1$ and $3 \mathrm{~Hz}$. Crack length was measured using a D.C. potential drop method. The current for the D.C. potential drop method was flowed for a short period, in order to minimize the disturbance of the electrochemical condition.

The load and the polarization current wave forms obtained at various crack propagation rates were recorded and stored in digital memories for the following analysis.

\section{Experimental Results}

\subsection{Tensile Test}

Figure 3 shows typical results of the relation between time and the tensile strain, and also the polarization current. The strain increased until a preset strain, and then was held. The polarization current was increased during the strain increasing, then it was decayed exponentially while the strain was held.

Total electric charge was obtained as an integration 


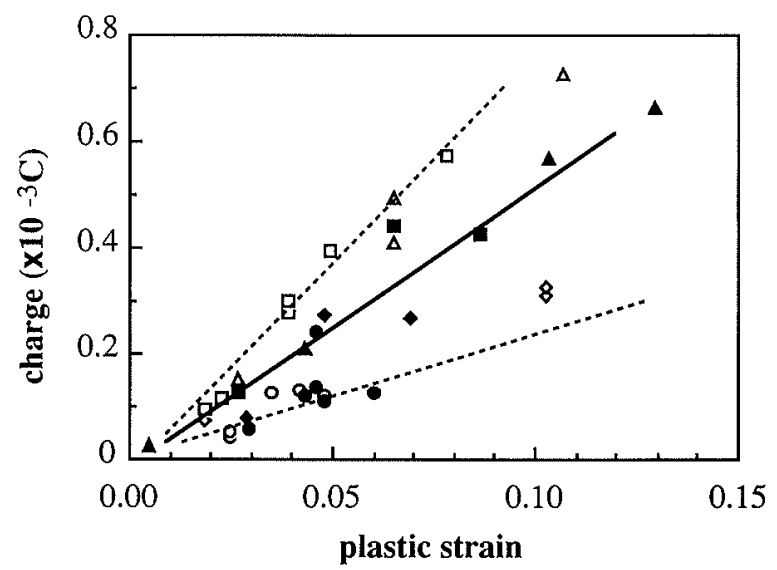

Fig. 4. Results of electric charge as a function of the plastic strain. Each symbol corresponds to the results measured on different specimen. Dotted lines indicate the data scattering range and the solid line shows the average value.

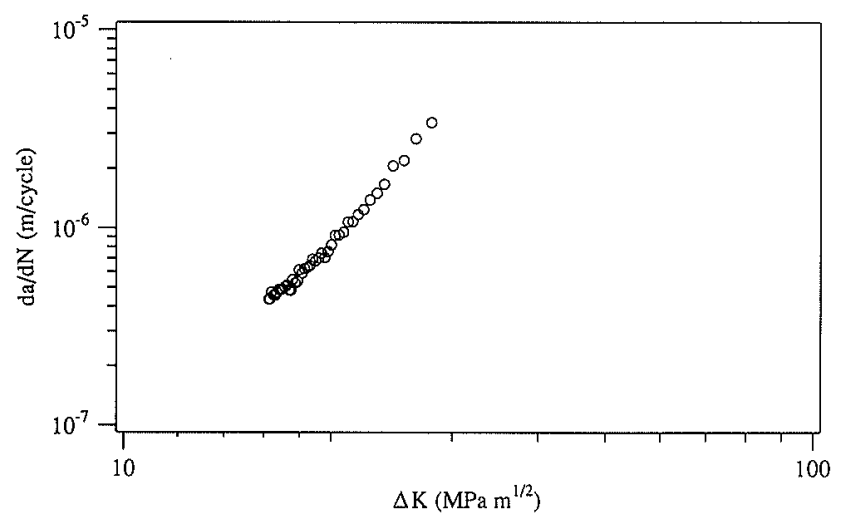

Fig. 5. Crack propagation rate $(d a / d N)$ vs. stress intensity factor range $(\Delta K)$ in $0.9 \% \mathrm{NaCl}$ aqueous solution at $0.1 \mathrm{~V}$ (vs. Ag/AgCl).

of the polarization current. The results are summarized in Fig. 4 as a function of the preset strain. The charge on each specimen increased linearly with increase in the strain, but the absolute magnitude was fluctuated.

\subsection{Fatigue Crack Propagation Test}

Figure 5 shows the relation between crack propagation rate $(d a / d N)$ and range of stress intensity factor $(\Delta K)$ in the environment. The electrochemical measurement was carried out in the range of Paris' law region $(\Delta K \approx 13$ to $30 \mathrm{MPa} \sqrt{\mathrm{m}}$ ). The exponent $m$ of Paris' law was approximately 4 .

Figure 6 shows the typical wave forms of the load and the polarization current observed during the crack propagation test $\left(d a / d N \approx 1 \times 10^{-6} \mathrm{~m} /\right.$ cycle $)$. An anodic polarization current peak was observed on increasing the load. It was decreased until load reached minimum, and then it increased slightly because of the reversed plastic deformation at the crack tip.

Since titanium is passivated in this environment, polarization current at steady state was approximately of the order of $0.1 \mu \mathrm{A}$ for the specimen used. However, this current is small enough compared with the polarization current shown in Fig. 6. Thus, the current during the fatigue crack propagation is mainly caused

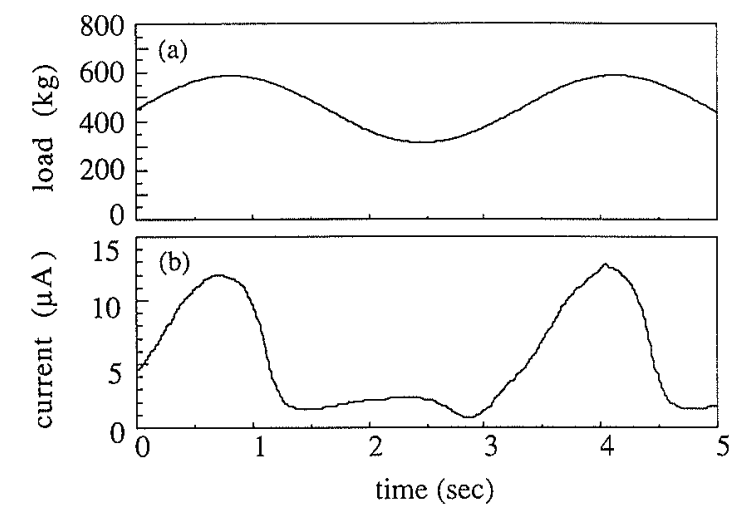

Fig. 6. Typical wave forms of the load (a) and polarization current (b) observed at the crack propagation rate of $\approx 1 \times 10^{-6} \mathrm{~m} /$ cycle.

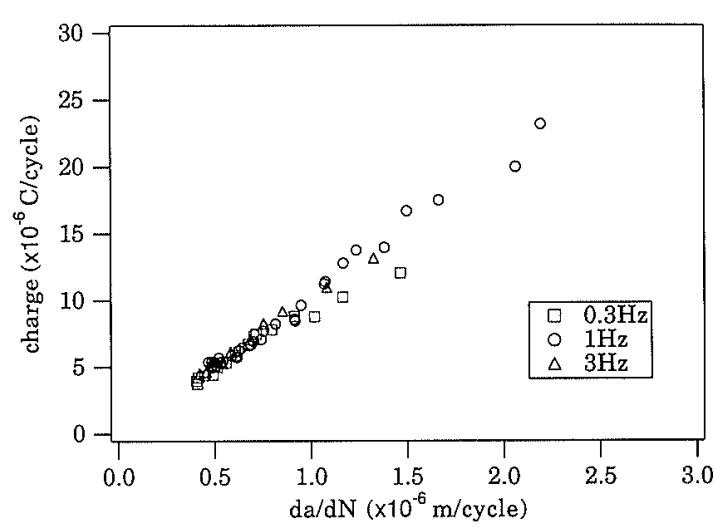

Fig. 7. Results of the electric charge passed during one loading cycle as a function of crack propagation rate $(d a / d N)$. Each symbol indicates the different loading frequency.

by the repassivation of fresh bare surface generated by the plastic deformation at the crack tip. Therefore, the electric charge passes for repassivation of the fresh surface which is required to propagate the corresponding crack propagation rate.

Therefore, the electric charge was calculated by the integration of the polarization current in one fatigue cycle and the electric charge for one fatigue cycle is summarized in Fig. 7 as a function of the crack propagation rate. This figure indicates that the charge of each cycle relates linearly to the crack propagation rate. Figure 7 also shows the result obtained at three loading frequencies of $0.3,1$ and $3 \mathrm{~Hz}$. Even if there was a 10 -fold change in the loading frequency, all the data fitted a straight line. Therefore it is suggested that when the crack propagation rate is the same at different frequencies, the charge is independent on the loading fequency, in other words, on the generation rate of the fresh surface. This relation certainly suggests that the fatigue crack propagation rate is linearly related to the amount of the fresh bare surface produced in each cycle at the crack tip.

\section{Discussion}

\subsection{Relationship between the Fresh Surface Area and the Electric Charge}

The tensile test results only showed the relation between the electric charge and strain, not the relation 


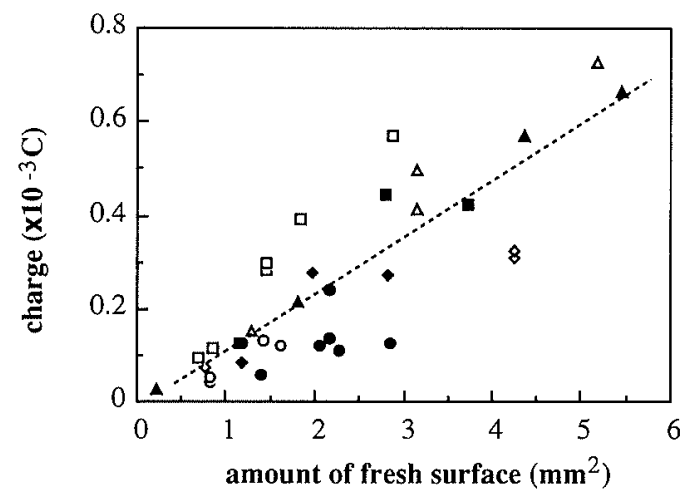

Fig. 8. Relation between the electric charge and calculated fresh surface area from the results showed in Fig. 4. Dotted line shows the average value.

between the charge and the fresh bare surface.

However, the area of fresh surface of tensile test is estimated from the applied plastic strain as follows. Assuming that a slip occurred on the maximum shear stressed plain angled of 45 degrees from the loading axis and that the slip direction was toward the tested surface, then the area of fresh surface due to the slip, $\Delta S_{t}$, is expressed as follows,

$$
\Delta S_{t}=B_{t} \Delta L \sqrt{2}
$$

where $B_{t}$ is the width of the tested surface, and $\Delta L$ is the increase in the length of the tested surface. The extension $\Delta L$ is

$$
\Delta L=L \varepsilon
$$

where $L$ is the length and $\varepsilon$ is the strain of the specimen measured by the strain gauge.

However, the specimen is poly-crystalline and each grain oriented randomly. Therefore, the slip occurred only in the grain oriented in higher Schmid factor slip systems. The slip systems are still angled at about 45 degrees to the loading axis, but the slip direction should be randomly scattered from the tested surface.

Under this assumption, the total area of slip steps generated on the front surface is calculated as follows,

$$
\Delta S_{t}=2 \sqrt{2} B_{t} L \varepsilon / \pi .
$$

The relation between the electric charge and the fresh bare surface area calculated in Eq. (3) is shown in Fig. 8. As described in the experimental results of the tensile test, the plots in the figure were scattered but those for each specimen were on a straight line. The tensile specimens were machined from the thick plate, and the crystallographic orientation of tested surface could not be identified. The material was annealed, but it is thought to be a slightly oriented texture. The scatter might be caused by the texture. However, Niwa, et al. reported that the total electric charge for a unit area of fresh bare surface is not affected by slip morphology. ${ }^{8)}$ According to the result, the relation between the fresh bare surface and the average electric charge required for repassivation of the surface is expressed experimentally as the following equation,

$$
\Delta S_{t}=8.3 \times\left. 10^{3} Q\left(\mathrm{~mm}^{2} / \mathrm{C}\right)\right|_{0.1 \mathrm{v}}
$$

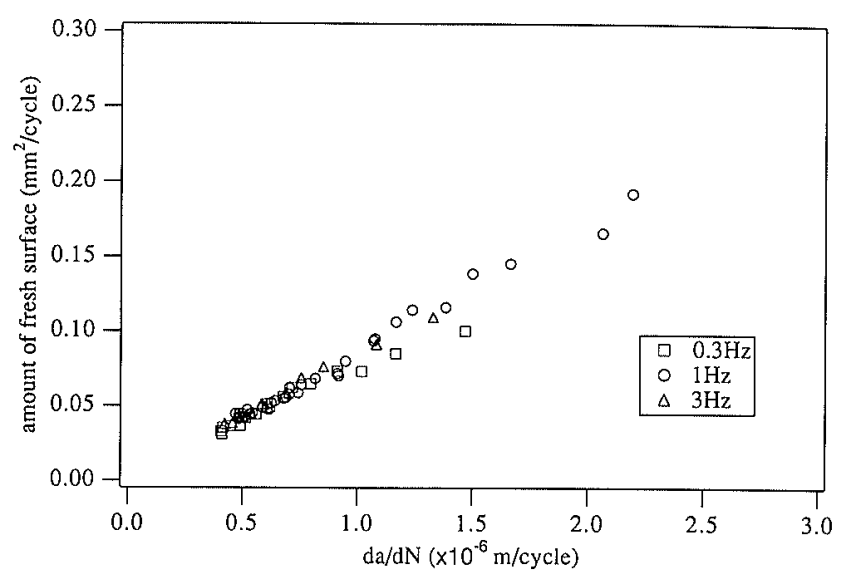

Fig. 9. Relation between $d a / d N$ and the fresh surface area produced during each fatigue cycle. Each symbol indicates the different loading frequency.

crack tip
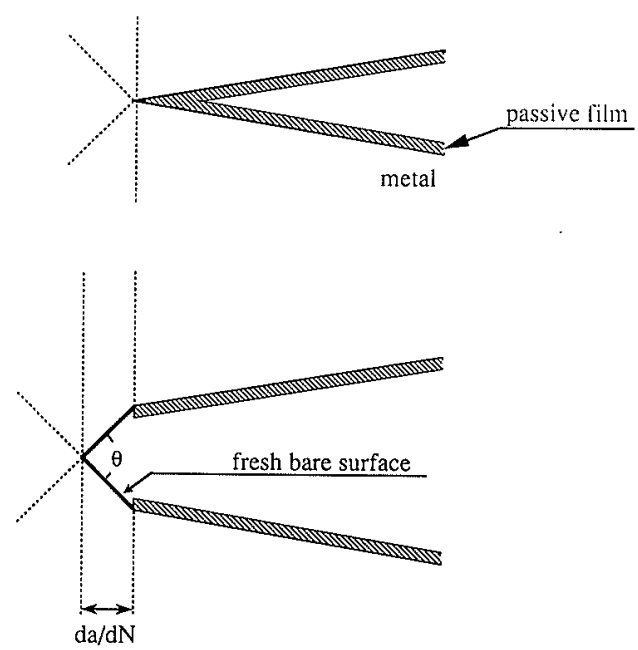

Fig. 10. Schematic drawings of the crack tip.

where $Q$ is the electric charge.

\subsection{Relation between Fresh Bare Surface at the Fatigue Crack Tip and $d a / d N$}

According to the results of crack propagation tests, the crack propagation rate relates linearly to the electric charge, and the relation between the area of the fresh bare surface and the electric charge was determined by the tensile tests. Therefore, we can now derive the relation between $d a / d N$ and the fresh bare surface produced by each fatigue cycle and this relation is shown in Fig. 9 which indicates that the fresh surface certainly corresponds to the $d a / d N$ linearly. The relation between the fresh bare surface area, $\Delta S_{p}$ and $\Delta K$ was concluded as follows,

$$
\Delta S_{p}=A(\Delta K)^{m}
$$

where $m$ corresponds to Paris' law exponent and $A$ is a constant. Now the relation between $\Delta S_{p}$ and $d a / d N$ will be discussed.

Figure 10 shows schematic drawings of the crack tip. $\Delta S_{p}$ can be calculated with the crack propagation rate, $d a / d N$, as a function of crack tip angle, $\theta$, assuming the shape of the crack tip as shown in Fig. 10, 


$$
\Delta S_{p}=2 B_{p} \frac{d a}{d N} \frac{1}{\cos (\theta / 2)}
$$

where $B_{p}$ is the thickness of the CT specimen.

From the result shown in Fig. 9, it is suggested that $\theta$ is approximately 140 degrees.

Kikukawa et $a .^{4\}}$ and Neumann et al. ${ }^{5)}$ observed the crack tip at near final fracture region with SEM, and found the $\theta$ is approximately 120 to 130 degrees on Fe-Si alloy. Therefore, it is considered that the results of 140 degrees of $\theta$ in this study is not an unreasonable value, even if different material was used.

According to Fig. 9, the fresh bare surface at the fatigue crack tip relates linearly to the crack propagation rate. This suggests that the crack tip angle $\theta$ is constant in the tested range of $d a / d N$, and crack tip shapes show the similar profile through the $\Delta K$ range.

Furthermore, it is known that the reversed plastic zone size at the front of the crack tip, $r_{p}$, is expressed as follows, ${ }^{9)}$

$$
r_{p}=B\left(\Delta K / \sigma_{y c}\right)^{2}
$$

where $B$ is a constant and $\sigma_{y c}$ is the cyclic yield stress. Then the reversed plastic zone size is proportional to the $(\Delta K)^{2}$. On the crack tip opening displacement, the opening is also the same sense as $r_{p}$.

On the other hand, the crack propagation rate is expressed as Paris' law, ${ }^{9)}$

$$
d a / d N=C(\Delta K)^{m} ; C, m \text { : constant }
$$

so that the crack propagation rate is proportional to the $(\Delta K)^{m}$.

The $m$-value varies with materials, and the value is in the range of approximately 2 to 6 for metals ${ }^{10)}$ and was approximately 4 in this study.

Therefore, it is concluded that the plastic zone size which gives the region of the plastic deformation at the front of the crack does not directly correspond to the fresh bare surface generated by the plastic deformation. This clarifies that the fatigue crack propagation mechanism directly relates to the fresh bare surface and not directly to the plastic zone size at the front of the crack, since the results obtained by fracture mechanics do not give any information on the fresh bare surface which directly causes crack propagation. Furthermore, the amount of plastic deformation at the crack tip occurred during cyclic loading strongly depends on the dislocation structure in the plastic zone and does not directly relates to the cyclic yield stress.

For further discussion, more detailed measurements of $\Delta S_{p}$ in lower fatigue crack propagation rate are required. However, the anodic passivation current is even small, and the precise dynamic measurement of the current is difficult at this moment.

\section{Conclusion}

Fatigue crack was propagated in pure titanium poly-crystal, and the amount of bare surface produced at the crack tip during one fatigue cycle was measured by the electrochemical method.

The amount of fresh bare surface at the fatigue crack tip $\left(\Delta S_{p}\right)$ relates linearly to the crack propagation rate as follows,

$$
\Delta S_{p}=A(\Delta K)^{m} \propto d a / d N
$$

where $m$ is the Paris' law exponent and $A$ is the constant.

This suggests that the shapes of crack tip show a similar profile through the tested $\Delta K$ range.

Consequently, it is clarified that the fatigue crack propagation mechanism directly relates to the fresh bare surface and not directly to the plastic zone size at the front of the crack.

\section{Acknowledgements}

The authors would like to thank Sumitorno Metal Industries, Ltd. for supplying the material. The technical assistance of Mr. Makoto Chujo at Tokyo Institute of Technology is also appreciated.

\section{REFERENCES}

1) C. Q. Bowles and D. Broek: Int. J. Fract. Mech., 8 (1972), No. 1,75 .

2) P. Neumann: Acta Metall., 22 (1974), 1155.

3) C. Q. Bowles and J. Schijve: Fatigue Mechanisms: Advances in Quantitative Measurement of Physical Damage, ASTM STP 811 , J. Lankford, D. L. Davidson, W. L. Morris and R. P. Wei, eds., ASTM, Philadelphia, PA, (1983), 400.

4) M. Kikukawa, M. Jono and M. Adachi: Fatigue Mechanisms, ASTM STP 675, J. T. Fong, ed., ASTM, Philadelphia, PA, (1979), 234.

5) P. Neumann, H. Fuhlrott and H. Vehoff: Fatigue Mechanisms, ASTM STP 675, J. T. Fong, ed., ASTM, Philadelphia, PA, (1979), 371 .

6) T. W. Crooker, F. D. Bogar and W. R. Cares: Corrosion-Fatigue Technology, ASTM STP 642, H. L. Craig, Jr., T. W. Crooker and D. W. Hoeppner, eds., ASTM, Philadelphia, PA, (1978), 189.

7) D. B. Dawson: Metall. Trans. A, 12A (1981), 791.

8) H. Niwa, S. Asawa, S. Haruyama and T. Mori: Corros. Sci., 23 (1983), No. 9, 959.

9) M. Klesnil and P. Lukas: Fatigue of Metallic Materials, Elsevier Scientific Publishing Co., Amsterdam, (1980), 81.

10) C. Hathias and R. M. Pelloux: Metall. Trans. A, 4A (1973), 1265. 